\title{
Acquired Factor V Inhibitor in a Patient with a Mechanical Aortic Valve under Warfarin Therapy
}

\author{
Tsukasa Nozu $^{1}$, Hiroaki Mita ${ }^{1}$ and Toshikatsu Okumura ${ }^{2}$
}

\begin{abstract}
An 84-year-old woman under warfarin therapy, who had undergone mechanical valve replacement 29 months previously, developed coagulation abnormalities after antibiotic treatment for pyelonephritis. Laboratory findings included PT at $47.6 \mathrm{sec}$, activated thromboplastin time (APTT) at $147 \mathrm{sec}$, factor V (FV) activity of $4 \%$ and FV inhibitor of $8 \mathrm{BU}$. Although overt bleeding was not observed, administration of prednisolone was started. Her coagulation abnormalities were rapidly normalized. It was later determined that the patient had received bovine thrombin at surgery. The presence of a FV inhibitor should be considered in the differential diagnosis in patients demonstrating an unexpected prolongation of PT under warfarin therapy following surgery.
\end{abstract}

Key words: factor $\mathrm{V}$ inhibitor, bovine thrombin, warfarin

(Inter Med 49: 2229-2233, 2010)

(DOI: 10.2169/internalmedicine.49.4042)

\section{Introduction}

Acquired factor V (FV) inhibitors have been documented since 1955 and about 150 cases with such inhibitors have been reported to date $(1,2)$. The most common cause of FV inhibitors is exposure to bovine thrombin which is used for achieving hemostasis particularly in cardiovascular surgery and neurosurgery (2). Bovine preparations are highly immunogenic, and patients who are treated with them may develop antibodies to bovine thrombin and other coagulation factors such as FV found in these preparations (3). There is approximately $70 \%$ homology between bovine and human coagulation factors, and these antibodies may cross-react with human coagulation factors leading to bleeding complications and/or an abnormal coagulation test $(4,5)$. Although a significant portion of the patients with $\mathrm{FV}$ inhibitors suffer bleeding complications, many cases are initially recognized because of abnormalities in common screening coagulation test such as in a prolongation of prothrombin time (PT) (2). Since some of the bleeding episodes are fatal (2), physicians should recognize this disease well.

We describe a patient with a history of bovine thrombin exposure who underwent mechanical aortic valve replace- ment and developed acquired FV inhibitor with a marked prolongation of PT under warfarin therapy. Since prolongation of PT is a common abnormality in both over-dose of warfarin and FV inhibitors, a prompt diagnosis of this disease for patients under warfarin therapy may be difficult. In this context, the presence of a FV inhibitor should be considered in the differential diagnosis of patients with an acquired postoperative coagulopathy. The purpose of this report is to present this rare disease and to enlighten physicians.

\section{Case Report}

An 84-year-old woman with Alzheimer's disease, congestive heart failure and chronic kidney disease was transferred and admitted to the convalescent ward in our hospital in August 2007 for the purpose of recuperation. She had undergone mechanical aortic valve replacement in April 2007 in another hospital because of aortic valve stenosis and had been under anticoagulation therapy using warfarin. She had no prior personal or family history of bleeding.

The clinical course is illustrated in Fig. 1. She became feverish because of pyelonephritis in mid-August 2009, and intravenous administration of ceftriaxone was started. Three

${ }^{1}$ Department of Internal Medicine, Shari General Hospital, Shari and ${ }^{2}$ Department of General Medicine, Asahikawa Medical College, Asahikawa Received for publication June 3, 2010; Accepted for publication July 22, 2010

Correspondence to Dr. Tsukasa Nozu, tnozu@ sea.plala.or.jp 


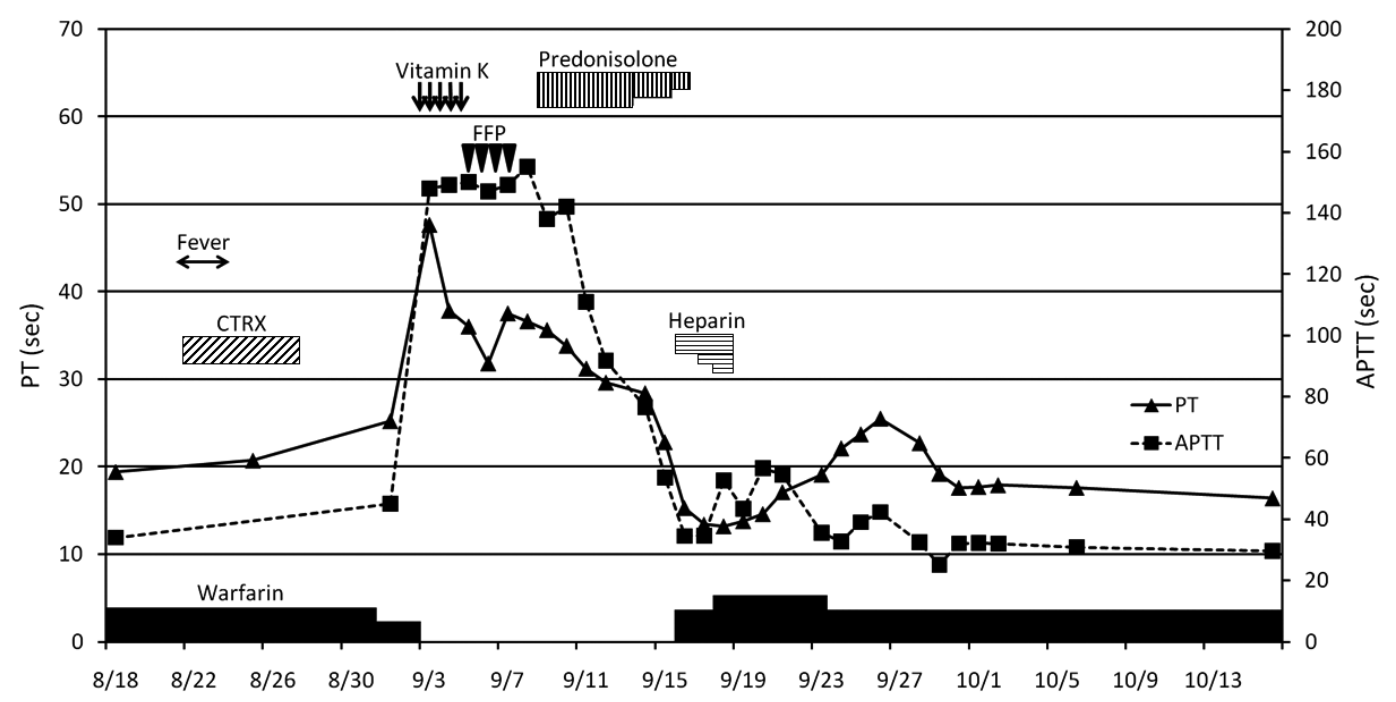

Figure 1. Clinical course. The coagulopathy with prolongation of PT and APTT occurred after antibiotic treatment for pyelonephritis. Neither administration of vitamin $\mathbf{K}$ nor fresh frozen plasma (FFP) ameliorated these abnormalities. However, prednisolone dramatically normalized these parameters. PT: prothrombin time, APTT: activated partial thromboplastin time, CTRX: ceftriaxone

Table 1. Laboratory Findings

\begin{tabular}{lll}
\hline Peripheral blood & Blood chemistry & Serology \\
WBC $11500 / \mu \mathrm{L}$ & TP $6.2 \mathrm{~g} / \mathrm{dL}$ & HBsAg $(-)$ \\
RBC $291 \times 10^{4} / \mu \mathrm{L}$ & Alb $3.5 \mathrm{~g} / \mathrm{dL}$ & HCV Ab (+) \\
Hb $9.7 \mathrm{~g} / \mathrm{dL}$ & AST $13 \mathrm{IU} / \mathrm{L}$ & ANA $\times 40$ \\
Ht $28.6 \%$ & ALT $14 \mathrm{IU} / \mathrm{L}$ & Anti-cardiolipin $\beta 2-\mathrm{GPI} \mathrm{Ab}$ \\
Plt $46.1 \times 10^{4} / \mu \mathrm{L}$ & LDH $174 \mathrm{IU} / \mathrm{L}$ & $<1.2 \mathrm{U} / \mathrm{mL}$ \\
& ALP133 IU/L & \\
Coagulation test & $\gamma$-GTP $12 \mathrm{IU} / \mathrm{L}$ & \\
PT $36.6 \mathrm{sec}$ & LAP $47 \mathrm{IU} / \mathrm{L}$ & \\
APTT 155 sec & CPK $24 \mathrm{IU} / \mathrm{L}$ & \\
TT $70 \%$ & T.Chol. $150 \mathrm{mg} / \mathrm{dL}$ & \\
HPT $75 \%$ & Na $139 \mathrm{mEq} / \mathrm{L}$ & \\
Fibrinogen $931 \mathrm{mg} / \mathrm{dL}$ & K $4.3 \mathrm{mEq} / \mathrm{L}$ & \\
FDP $1.5 \mu \mathrm{mL}$ & Cl $100 \mathrm{mEq} / \mathrm{L}$ & \\
AT $132 \%$ & BUN $37.8 \mathrm{mg} / \mathrm{dL}$ & \\
Coagulation time 19, & Cre $1.48 \mathrm{mg} / \mathrm{dL}$ & \\
& CRP $1.14 \mathrm{mg} / \mathrm{dL}$ & \\
\hline
\end{tabular}

ANA, anti-nuclear antibody; Anti-cardiolipin $\beta 2$-GPI Ab, anti-cardiolipin

$\beta 2$-glycoprotein I complex antibody.

days later, she was afebrile and prothrombin timeinternational normalized ratio (PT-INR) was 2.38 (PT 20.7 $\mathrm{sec})$ at the dose of $1.75 \mathrm{mg}$ of warfarin. Her inflammatory reaction became almost negative 7 days later and ceftriaxone was stopped. PT-INR was 3.41 (PT $25.2 \mathrm{sec}$ ) at the beginning of September and then the dose of warfarin was reduced to $1.5 \mathrm{mg}$. Two days later, she was symptomless but PT-INR was increased to 10.8 (PT $47.6 \mathrm{sec}$ ) and activated thromboplastin time (APTT $147 \mathrm{sec)}$ was also markedly prolonged. Then warfarin was stopped and vitamin $\mathrm{K}$ was intravenously administered. In spite of these treatments, PT-
INR was not dramatically changed and it fluctuated between 5 and 7. Moreover, administration of fresh frozen plasma (FFP) did not ameliorate PT-INR either. Laboratory findings on early September are summarized in Table 1. Coagulation studies revealed that PT, APTT and coagulation time were markedly prolonged, but hepaplastin test was normal, suggesting possibly reduced activity of FV. Anti-nuclear antibody was $\times 40$, and anti-cardiolipin $\beta 2$-glycoprotein I complex antibody was negative (normal range <3.5). Plasma mixing test using a non-incubated mixture of patient and normal plasma $(1: 1)$ demonstrated that APTT was consid- 


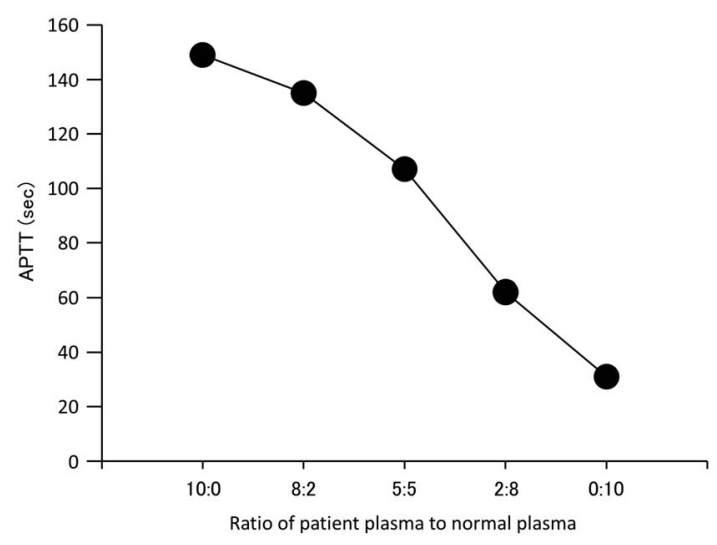

Figure 2. Cross mixing test utilized a mixture of patient and normal plasma at various ratios after incubation for 2 hours at $37^{\circ} \mathrm{C}$. Titration curve indicated not deficiency but a typical pattern of existence of coagulation factor inhibitors.

erably corrected. Then mixing test after incubation for 2 hours at $37^{\circ} \mathrm{C}$ with mixtures at various ratios was performed (Fig. 2). It demonstrated not deficiency but a typical pattern of existence of coagulation factor inhibitors and also suggested that the inhibitors were a delayed type of action. Coagulation factor assays showed a significant decrease in the FV activity (Table 2). The specific inhibitor for FV was also positive (Table 2). On the basis of the above findings, she was diagnosed as having coagulation abnormalities induced by a FV inhibitor. At this time, the evidence of bovine thrombin exposure at the cardiac surgery was disclosed by inquiring her surgeons in the previous hospital. Although overt bleeding was not observed, administration of prednisolone $(0.8 \mathrm{mg} / \mathrm{kg} / \mathrm{day})$ was started on September 9. Coagulation studies revealed remarkable recovery and on September 16, warfarin was restarted together with heparin. Prednisolone was tapered and stopped on September 17. Coagulation factor assays on September 16 showed that FV activity was normalized and an inhibitor for FV was negative (Table 2). After that, warfarin therapy was continued and marked variations of PT-INR were not observed. She was hospitalized for 8 months and an adequate PT-INR level was obtained by $1.5-2 \mathrm{mg}$ of warfarin.

\section{Discussion}

The most common cause of FV inhibitors is exposure to bovine thrombin (iatrogenic FV inhibitors). Two-thirds of the reported cases had a history of such exposure (2). On the other hand, spontaneous FV inhibitors have also been reported and surgical procedures, infections or exposure to antibiotics precede the inhibitor development in these patients (2). Since the present case had a history of bovine thrombin exposure, it might be an iatrogenic case. However, FV inhibitors emerged 29 months after the exposure, which is quite uncommon. It was reported that bovine thrombinassociated inhibitors emerge a mean of 8.3 days after exposure (2). On the other hand, infection and antibiotic expo-
Table 2. Coagulation Factor Assays

\begin{tabular}{lcc} 
& September 8 & September 16 \\
\hline Factor II & $60 \%$ & $106 \%$ \\
Factor V & $4 \%$ & $61 \%$ \\
Factor VII & $59 \%$ & $98 \%$ \\
Factor VIII & $131 \%$ & $>200 \%$ \\
Factor IX & $134 \%$ & $150 \%$ \\
Factor X & $54 \%$ & $104 \%$ \\
Factor XI & $123 \%$ & $120 \%$ \\
Factor XII & $55 \%$ & $73 \%$ \\
Factor XIII & $144 \%$ & $131 \%$ \\
\hline Factor V inhibitor & $8 \mathrm{BU}$ & $0 \mathrm{BU}$ \\
\hline
\end{tabular}

BU, Bethesda units.

sure preceded the emergence of inhibitor in the present case, which is a common feature of spontaneous FV inhibitors. These findings indicate that the present case had the features both iatrogenic and spontaneous cases. Since we did not prove the immunoglobulin binding to bovine thrombin or FV in vitro in the present case, it is unknown whether or not bovine thrombin exposure was truly associated with the development of FV inhibitor in this patient. There have been no reports demonstrating that $\mathrm{FV}$ inhibitors emerge such a long time after the exposure as in the present case, but it was reported that antibodies against bovine proteins possibly persist for more than 10 years after the exposure (3). In this context, the possibility of this association cannot be denied in our case. It is conceivable that the present case was primed by potent antigen exposure (bovine thrombin), and infection and antibiotics may have been the trigger to induce further activation of the immune system leading to a significant production of antibodies against $\mathrm{FV}$.

Patients with FV inhibitors are diagnosed by an abnormal coagulation test such as a prolongation of PT or APTT performed by chance in the asymptomatic cases. The present case under warfarin therapy with a prolongation of PT was initially diagnosed as warfarin over-dose. Since marked variations of PT-INR are frequently observed in elderly patients under warfarin therapy particularly associated with appetite loss or infections (6), our initial decision seemed to be acceptable. However, in spite of administration of vitamin $\mathrm{K}$ or FFP, the coagulation abnormality did not ameliorate, and thus warfarin over-dose could be denied. In addition to these results, the hepaplastin test was normal, suggesting that the coagulopathy was not induced by warfarin but possibly by reduced activity of FV. Then we performed cross-mixing test demonstrating the existence of coagulation factor inhibitors, and at this time upon inquiry with her surgeon, we became aware that she had a history of bovine thrombin exposure 29 months previously. Definitive diagnosis was made by the result of coagulation factor assays and positivity for specific inhibitor for FV. 
Although her PT, APTT and coagulation time were markedly prolonged, overt bleeding was not detected. A previous study demonstrated that a significant portion of these patients suffered from bleeding complications (2). Seventy-two percent of the reported cases of spontaneous FV inhibitors suffered bleeding complications with 17 percent of these episodes being fatal. On the other hand, 33 percent of bovine thrombin-induced inhibitor patients developed bleeding, which was fatal in 6 percent. These lines of evidence suggest that spontaneous FV inhibitors appear to be associated with more bleeding and severe bleeding episodes than iatrogenic inhibitors, but the reason for this remains known. On the other hand, some cases were reported to be self-limiting, i.e. inhibitors disappeared without any treatment after several months $(2,7)$. The management strategy for this disease with evidence including treatment or no treatment, has not been determined to date, therefore we decided to treat her because the mortality of bleeding cases was reported to be high (7). Moreover, she had several comorbidities such as congestive heart failure and chronic kidney disease, suggesting that bleeding may be a fatal complication for her. A previous study demonstrated that coagulation tests such as PT, FV activity or inhibitor titer are of little help in identifying which patients are likely to bleed (2), indicating her probability of bleeding was not estimated. We used prednisolone following the anecdotal treatment experiences of other investigators $(2,8)$. We judged that the benefit of prednisolone administration surpassed its drawbacks, but we do not know whether this judgment was correct. In any event, her coagulation abnormalities were rapidly improved after beginning this treatment. However, there is a possibility that this rapid amelioration resulted from not the effect of prednisolone but rather from the self-limiting disease. Streiff and Ness reviewed the treatment of patients with FV inhibitors and recommended steroid therapy as an initial trial because of the higher efficacy of corticosteroids with $74 \%$ success (2). In addition to corticosteroids, cyclosporine A, cytotoxic chemotherapy agents, intravenous immunoglobulins and reduction of antibody titers with plasmapheresis were also reported to be effective for FV inhibitors (9-11).

The most important point of the present case is that FV inhibitors emerged after an extremely long period, i.e. 29 months, from the bovine thrombin exposure. As mentioned above, we did not demonstrate a direct association between bovine thrombin exposure and FV inhibitors in the present case, but we could not deny its possibility. If this hypothesis is true, FV inhibitors may develop at any time during lifetime in patients with a history of its exposure. Fortunately, we could know the history of our patient, but confirmation of its exposure can be difficult several years after surgery. Bovine thrombin exposure is common particularly among patients who underwent cardiac surgery including mechanical valve replacement (2). Patients with a mechanical valve need warfarin therapy throughout lifetime and PT is monitored regularly in order to determine the optimal dose of warfarin. Since a prolongation of PT is a common abnor- mality in both an over-dose of warfarin and FV inhibitors, prompt and accurate diagnosis of this disease in the patients under warfarin therapy may be difficult. In this context, the presence of FV inhibitors should be considered in the differential diagnosis in any patient with an acquired postoperative coagulopathy, even though several years passed after surgery. Although the number of FV inhibitors reported remains small, a high frequency of developing FV inhibitors after bovine thrombin exposure has been reported. Banninger et al found that $42 \%$ of cardiac surgery and $20 \%$ of neurosurgery patients developed FV inhibitors after bovine thrombin exposure (12), suggesting a large number of FV inhibitor cases may be unrecognized. Because some cases develop fatal bleeding, physicians should know this disease well and prompt diagnosis is needed.

In summary, we presented a case with a FV inhibitor, which developed after a long period from exposure to bovine thrombin. Since patients who have undergone mechanical valve replacement may have a history of bovine thrombin exposure, physicians should consider not only over-dose of warfarin but also the possibility of FV inhibitors in these patients with a prolongation of PT. Many FV inhibitor cases are thought to be misdiagnosed, physicians should be alerted to this disease in order to prevent the fatal bleeding comorbidity.

\section{References}

1. Leus B, Devreese K, van den Bossche J, Malfait R. Factor V inhibitor: case report. Blood Coagul Fibrinolysis 17: 585-587, 2006.

2. Streiff MB, Ness PM. Acquired FV inhibitors: a needless iatrogenic complication of bovine thrombin exposure. Transfusion $\mathbf{4 2}$ : 18-26, 2002.

3. Ortel TL, Mercer MC, Thames EH, Moore KD, Lawson JH. Immunologic impact and clinical outcomes after surgical exposure to bovine thrombin. Ann Surg 233: 88-96, 2001.

4. Schoenecker JG, Johnson RK, Fields RC, et al. Relative purity of thrombin-based hemostatic agents used in surgery. J Am Coll Surg 197: 580-590, 2003.

5. Nesheim ME, Nichols WL, Cole TL, et al. Isolation and study of an acquired inhibitor of human coagulation factor V. J Clin Invest 77: 405-415, 1986.

6. Saito R, Akao H, Kaseno K, et al. Marked PT-INR prolongation associated with appetite loss due to urinary tract infection in a late elderly case with chronic atrial fibrillation. Nippon Ronen Igakkai Zasshi 46: 541-544, 2009 (in Japanese, Abstract in English).

7. Ang $\mathrm{AL}$, Kuperan $\mathrm{P}, \mathrm{Ng} \mathrm{CH}, \mathrm{Ng} \mathrm{HJ}$. Acquired factor $\mathrm{V}$ inhibitor. A problem-based systematic review. Thromb Haemost 101: 852859, 2009.

8. Endo H, Kawauchi K, Tomimatsu M, et al. Acquired factor V inhibitor responsive to corticosteroids in a patient with double cancers. Intern Med 46: 621-625, 2007.

9. Chong LL, Wong YC. A case of factor V inhibitor. Am J Hematol 19: 395-399, 1985.

10. de Raucourt E, Barbier C, Sinda P, Dib M, Peltier JY, Ternisien C. High-dose intravenous immunoglobulin treatment in two patients with acquired factor V inhibitors. Am J Hematol 74: 187-190, 2003.

11. Fu YX, Kaufman R, Rudolph AE, Collum SE, Blinder MA. Multimodality therapy of an acquired factor $\mathrm{V}$ inhibitor. Am J Hematol 51: 315-318, 1996.

12. Banninger H, Hardegger $\mathrm{T}$, Tobler A, et al. Fibrin glue in surgery: 
Inter Med 49: 2229-2233, 2010 DOI: 10.2169/internalmedicine.49.4042

frequent development of inhibitors of bovine thrombin and human

factor V. Br J Haematol 85: 528-532, 1993.

(C) 2010 The Japanese Society of Internal Medicine http://www.naika.or.jp/imindex.html 